\title{
Recomendaciones dietéticas para pacientes con Enfermedad Renal Crónica e infección por SARS-CoV-2
}

\author{
Almudena Pérez-Torres ${ }^{1,2,3}$, Alberto Caverni-Muñoz ${ }^{3,4}$, Filo Trocoli-González ${ }^{1,3}$, Ana Sanjurjo-Amado ${ }^{3,5}$, \\ Guillermina Barril-Cuadrado $0^{3,6}$ \\ ${ }^{1}$ Servicio de Nefrología. Hospital Universitario La Paz. IdiPaz. Madrid. España \\ 2 Unidad de Nutrición. Hospital Universitario Santa Cristina. Madrid. España \\ ${ }^{3}$ Miembro del Grupo de Nutrición de la Sociedad Española de Nefrología. Madrid. España \\ ${ }^{4}$ Servicio de dietética. Asociación de Enfermos Renales. Alcer Ebro. Zaragoza. España \\ ${ }^{5}$ Servicio de Nefrología. Hospital Da Costa Burela. Lugo. España \\ ${ }^{6}$ Servicio de Nefrología. Hospital Universitario La Princesa. Madrid. España
}

Como citar este artículo:

Pérez-Torres A, Caverni Muñoz A, Trocoli-Gonzalez F, Sanjurjo-Amado A, Barril-Cuadrado G. Recomendaciones dietéticas para pacientes con Enfermedad Renal Crónica e infección por SARS-Cov-2.

Enferm Nefrol. 2020 Jul-Sep;23(3):244-251

\section{Resumen}

La prevalencia de malnutrición en paciente con Enfermedad Renal Crónica es elevada, aumentando en pacientes con infección por SARS-CoV-2. La relación existente entre inflamación y nutrición es conocida en la enfermedad renal, por lo que la presencia previa de cuadros de malnutrición empeora el pronóstico de la infección.

El objetivo del presente artículo es la creación de recomendaciones dietéticas específicas para pacientes con enfermedad renal crónica e infección o post- infección por SARS-CoV-2, adaptadas al estadio de enfermedad y a la etapa del proceso de infección.

El abordaje nutricional comienza por la valoración del estado nutricional, para lo que se recomiendan minimizar el contacto físico mediante la utilización de los criterios Global Leadership Initiative on Malnutrition (GLIM), y el cuestionario rápido de sarcopenia (SARC-F).

Las recomendaciones dietéticas deben considerar el estadio de enfermedad renal crónica, la etapa de infección por SARS-CoV-2 y las complicaciones surgidas

\section{Correspondencia:}

Almudena Pérez Torres e-mail: aperezt@salud.madrid.org que comprometan la ingesta oral, entre las más comunes se encuentran: anorexia, ageusia, disfagia y diarrea. En el presente documento se han elaborado tablas de raciones de ingestas diarias adaptadas a las diferentes situaciones. En aquellos pacientes que no cubran los requerimientos nutricionales se recomienda comenzar con la suplementación nutricional de manera precoz, considerando las consecuencias de la infección descrita.

Debido al elevado riesgo de malnutrición en pacientes con enfermedad renal cónica e infección por SARSCoV-2, se recomienda la adaptación de la valoración del estado nutricional y su tratamiento, así como realizar una monitorización tras la fase de infección activa.

PALABRAS CLAVE: COVID-19; SARS-CoV-2; enfermedad renal crónica; diálisis; nutrición; dieta.

\section{Dietary recommendations for patients with Chronic Kidney Disease and SARS-CoV-2 infection}

\section{Abstract}

The prevalence of malnutrition in patients with Chronic Kidney Disease is high, increasing in patients with 
SARS-CoV-2 infection. The relationship between inflammation and nutrition in kidney disease is known, so the previous presence of malnutrition conditions worsens the prognosis of infection.

The objective of this article is the creation of specific dietary recommendations for patients with chronic kidney disease and infection or post-infection by the SARS-CoV-2 virus, adapted to the stage of the disease and the stage of the infection process.

The nutritional approach begins with the assessment of nutritional status, recommending minimizing physical contact through the use of the Global Leadership Initiative on Malnutrition (GLIM) criteria and the rapid sarcopenia questionnaire (SARC-F).

The dietary recommendations should consider the stage of chronic kidney disease, the stage of infection by SARS-CoV-2 and the complications arising that compromise oral intake, among the most common are: anorexia, ageusia, dysphagia and diarrhea. In this document, tables of daily intakes have been prepared adapted to different situations. In those patients who do not meet the nutritional requirements, it is recommended to start with an early nutritional supplementation, considering the consequences of the infection described.

Due to the high risk of malnutrition in patients with chronic kidney disease and SARS-CoV-2 infection, it is recommended to adapt the assessment of nutritional status and treatment, as well as to carry out monitoring after the active infection phase.

KEY WORDS: COVID-19; SARS-CoV-2; chronic kidney disease; dialysis; nutritrion; dietary.

\section{Introducción}

La infección por SARS-CoV-2 plantea un gran desafío para salud mundial, constituyendo un factor de riesgo independiente de mortalidad hospitalaria ${ }^{1,2}$. Actualmente sabemos, que el espectro clínico de la infección varía desde una forma asintomática, que cursa como una enfermedad leve con signos y síntomas inespecíficos de afectación respiratoria aguda hasta una neumonía grave con insuficiencia respiratoria y shock séptico ${ }^{3,4}$. La infección se clasifica en diferentes etapas: leve, moderada o grave según la sintomatología.
Los estudios recientes plantean la presencia de desnutrición en un $50 \%$ de los pacientes con infección por SARS-CoV-2, debido principalmente al hipercatabolismo secundario a la inflamación producida por el proceso de infección, y a los efectos secundarios descritos en los pacientes, como son, la disminución de la ingesta por la presencia de anorexia, disfagia o disnea, y a la disminución de la absorción por procesos diarreicos ${ }^{5}$.

Hasta la fecha no se ha realizado ningún estudio específico en población con Enfermedad Renal Crónica (ERC), pero debido a la elevada prevalencia de malnutrición en este colectivo de pacientes y a los efectos secundarios relacionados con el proceso de infección por SARS-CoV-2, el objetivo principal del presente artículo es la creación de recomendaciones dietéticas específicas para pacientes con ERC e infección o post- infección por SARS-CoV-2 adaptadas al estadio de enfermedad y a la etapa del proceso de infección.

\section{Valoración nutricional en paciente con ERC e in- fección por SARS-CoV-2}

Una de las principales limitaciones en la realización de la valoración nutricional en estos pacientes, es el contacto físico, por lo que se proponen como principales herramientas para la valoración nutricional: la realización de un método de cribado que evalúe la pérdida involuntaria de peso y la ingesta dietética, como puede ser el Malnutrition Universal Screening Tools (MUST) ${ }^{6}$, y si el paciente da positivo aplicar los criterios Global Leadership Initiative on Malnutrition (GLIM) ${ }^{7}$ de valoración nutricional en los que la necesidad de contacto físico es mínimo. Debido a la elevada prevalencia de sarcopenia en el pacientes renal y en los pacientes con infección por SARS-CoV-2, se recomienda incluir el cuestionario rápido de sarcopenia (SARC-F) ${ }^{8}$.

\section{Recomendaciones dietéticas}

La intervención nutricional debe empezar tras la valoración del estado nutricional, y comenzar por el tratamiento dietético siempre que la vía oral se encuentre disponible. En la Tabla 1, se muestra el tamaño de ración de los diferentes grupos ${ }^{9}$ de alimentos, los cuales se irán adaptando según fase de infección por SARS-CoV-2 y estadio de la ERC.

Todas las raciones de ingestas recomendadas se han elaborado adaptando las recomendaciones de las diferentes sociedades científicas ${ }^{10,11,12,13}$. 
Tabla 1. Definición del peso y tamaño de ración según los diferentes grupos de alimentos.

\begin{tabular}{|c|c|c|}
\hline Grupos alimentos & Tamaño de la ración (crudo y neto) & Medidas caseras \\
\hline $\begin{array}{l}\text { Cereales (pan, pasta, arroz, } \\
\text { patata y otros cereales) }\end{array}$ & $\begin{array}{l}\text { Pan: } 30-60 \mathrm{~g} \\
\text { Arroz o pasta: } 50-80 \mathrm{~g} \text { crudo } \\
\text { 150-200 cocido } \\
\text { Cereales desayuno: } 20-40 \mathrm{~g} \\
\text { Patatas: } 150-200 \mathrm{~g}\end{array}$ & $\begin{array}{l}1-2 \text { trozos de } 4 \text { dedos de grosor } \\
1 \text { plato hondo normal } \\
2-3 \text { cucharadas soperas } \\
1 \text { patata mediana ó } 2 \text { pequeñas }\end{array}$ \\
\hline Verduras y hortalizas & $150-250 \mathrm{~g}$ & $\begin{array}{l}1 \text { plato hondo de ensalada variada } \\
1 \text { plato hondo de verdura cocida } \\
1 \text { tomate grande }\end{array}$ \\
\hline Frutas & $150-200 \mathrm{~g}$ & $\begin{array}{l}1 \text { pieza mediana, } 2 \text { mandarinas pequeñas, } \\
3 \text { ciruelas, } 1 \text { rodaja de melón, } 1 \text { taza de } \\
\text { cerezas o fresas }\end{array}$ \\
\hline Lácteos & $\begin{array}{l}\text { Leche: } 200-250 \mathrm{ml} \\
\text { Yogur: } 2 \text { x } 125 \mathrm{ml} \\
\text { Queso curado/semicurado: } 40-60 \mathrm{~g} \\
\text { Queso fresco: } 80-125 \mathrm{~g}\end{array}$ & $\begin{array}{l}1 \text { vaso de leche } \\
2 \text { yogures } \\
2-3 \text { cuñas de queso } \\
1 \text { tarrina individual }\end{array}$ \\
\hline Aceite de Oliva & $10 \mathrm{ml}$ & 1 cucharada mediana \\
\hline Legumbres & $\begin{array}{l}60-80 \text { g crudo } \\
150-200 \text { g cocido }\end{array}$ & $\begin{array}{l}1 \text { plato hondo normal } \\
2-3 \text { cazos pequeños con caldo }\end{array}$ \\
\hline Pescado & $100-150 \mathrm{~g}$ & $\begin{array}{l}1 \text { filete mediano, } 1 \text { pieza de ración mediana, } \\
1 \text { rodaja mediana }\end{array}$ \\
\hline Huevos & $65-100 \mathrm{~g}$ & $\begin{array}{l}1 \text { huevo grande, } 2 \text { huevos pequeños } 0 \\
\text { medianos }\end{array}$ \\
\hline Carnes & $100-150 \mathrm{~g}$ & $\begin{array}{l}1 \text { filete mediano } \\
1 \text { muslo de pollo mediano } \\
1 \text { pechuga }\end{array}$ \\
\hline Frutos secos & $20-30 \mathrm{~g}$ & 1 puñado sin cáscara \\
\hline
\end{tabular}

\section{Recomendaciones dietéticas para pacientes con ERC e infección leve por SARS-CoV-2}

La Tabla 2 muestra las raciones recomendadas para pacientes con ERC e infección leve por SARS-CoV-2, adaptadas a la etapa de la enfermedad en la que se encuentren.

Las recomendaciones generales en esta etapa de la infección por SARS-CoV-2 son:

- Asegurar ingesta hídrica. A través de agua, caldos vegetales, caldos de carne o pescado, o infusiones. La recomendación para población general de ingesta de líquido es de 2-2,5 litros, pero dada la alta prevalencia de retención hídrica en el paciente renal se deja a criterio del clínico ${ }^{14}$.

- Ingerir 5 raciones de frutas y verduras al día frescas y de temporada. En caso de hiperpotasemia, seguir las recomendaciones para el control de potasio.
- Asegurar el consumo diario de cereales preferiblemente integral, a excepción de hiperfosfatemias e hiperpotasemias, que se recomiendan refinados.

- Se recomienda una ingesta de lácteos diaria preferiblemente seleccionar lácteos fortificadas en vitamina D. Elegir variedad de leche semidesnatada y queso semicurado. Asegurar el consumo de 1 yogur al día, incluyendo el suero lácteo.

- Consumo diario de carnes, pescado, huevos o legumbres según la siguiente distribución:

- Carnes magras o blancas: 3-4 veces a la semana.

- Pescados: 4-5 veces a la semana. Asegurar el consumo de pescado azul al menos 2 veces por semana.

- Huevos: 3-4 veces a la semana; preferiblemente en tortilla, plancha o pasados por agua. 
Tabla 2. Raciones diarias de ingesta recomendadas según estadio de la Enfermedad Renal Crónica en pacientes con infección leve por SARS-CoV-2.

\begin{tabular}{|c|c|c|c|c|c|}
\hline Grupo de alimentos & ERCA 1-2 & ERCA 3-5 sin diálisis & Hemodiálisis & Diálisis Peritoneal & Trasplante \\
\hline Cereales (rac/día) & 5 & 6 & 5 & 4 & 5 \\
\hline $\begin{array}{l}\text { Verduras y hortalizas } \\
\text { (rac/día) }\end{array}$ & 2 & 2 & 2 & 2 & 2 \\
\hline Frutas (rac/día) & 3 & 2 & 2 & $2-3$ & 3 \\
\hline Lácteos (rac/día) & 1,5 & 1,5 & 1 & 1 & 1,5 \\
\hline Aceite de Oliva (rac/día) & 6 & 6 & 6 & 7 & 6 \\
\hline $\begin{array}{l}\text { Carnes, pescados, } \\
\text { huevos y legumbres } \\
\text { (rac/día) }\end{array}$ & 1 & 1 & 2 & 3 & 1 \\
\hline Frutos secos (rac/día) & 1 & Individualizar & No recomendado & Individualizar & 1 \\
\hline Azúcares & Muy moderado & Muy moderado & Muy moderado & No recomendado & Muy moderado \\
\hline $\begin{array}{l}\text { Dulces, Bollería, } \\
\text { grasas untables y } \\
\text { snacks salados }\end{array}$ & Ocasional & Ocasional & Ocasional & No recomendado & Ocasional \\
\hline
\end{tabular}

Nota: Las raciones han sido calculadas para un peso estimado de $70 \mathrm{~kg}$. Abreviaturas: rac/día, ración/día.

- Legumbres: 2-3 veces por semana. Si consume legumbre en conserva debe eliminar el líquido de la conserva.

- El consumo de frutos secos está permitido dependiente de la fase de la ERC, siempre crudos o tostados sin sal y sin azúcar.

- Priorizar el consumo de aceite oliva virgen extra, tanto para cocinar como para aliño.

- Evitar el consumo de alimentos ultraprocesados y de comida rápida.

\section{Recomendaciones dietéticas para pacientes con ERC e infección moderada por SARS-CoV-2}

Durante las fases moderada y grave, la presencia de inflamación es mayor creando un estado de hipercatabolismo, por lo que se recomienda ajustar los requerimientos proteicos. En la Tabla 3 se muestran las raciones de alimentos recomendadas según etapa de la ERC, y ajustándose a la presencia de hipercatabolismo.

Los principales síntomas descritos en esta fase de infección por SARS-CoV-2 son la presencia de disfagia y disnea, anorexia, hipogeusia, y diarrea secundaria principalmente al tratamiento farmacológico prescrito. En esta fase las principales recomendaciones dietéticas son:

- Asegurar el consumo de todas las raciones indicadas fraccionando la ingesta en 5-6 tomas al día, y si es preciso, aumentar el número de tomas.
- Elegir variedad de leche entera y quesos grasos para aumentar el aporte calórico. Asegurar el consumo de 1-2 yogur enteros al día, incluyendo el suero lácteo ${ }^{15}$.

- Imprescindible consumo diario de carnes, pescado, huevos o legumbres en comida, cena y una toma extra en desayuno, media mañana o merienda.

- En el caso en el que se manifieste disnea o disfagia, se recomienda modificar la textura y consistencia de la dieta, a dietas: blandas, fácil masticación o túrmix.

- Ante la presencia de anorexia, fraccionar la ingestas y enriquecer la dieta.

- En el caso de diarrea, asegurar ingesta hídrica y realizar dieta astringente o de protección gástrica.

- Si existe presencia de hipogeusia, utilizar potenciadores del sabor tipo especias.

Como medidas complementarias se recomienda, en el caso en el que el paciente se encuentre hospitalizado ${ }^{16}$ :

- Pesar siempre que sea posible para control de peso.

- Control de ingesta liquida y diuresis para hacer balance líquido.

- No interrumpir la oxigenoterapia durante las ingestas.

- Elevar el cabecero de la cama en un ángulo $\geq 30^{\circ}$ para prevenir el riesgo de aspiración. 
Tabla 3. Raciones diarias de ingesta recomendadas según estadio de la Enfermedad Renal Crónica en pacientes con infección moderada por SARS-CoV-2.

\begin{tabular}{|c|c|c|c|c|c|}
\hline Grupo de alimentos & ERCA 1-2 & ERCA 3-5 sin diálisis & Hemodiálisis & Diálisis Peritoneal & Trasplante \\
\hline Cereales (rac/día) & 6 & $\begin{array}{c}6 \\
\text { En paciente } \\
\text { hipercatabólico: } 7\end{array}$ & 6 & 5 & 6 \\
\hline Verduras y hortalizas (rac/día) & 2 & 2 & 2 & 2 & 2 \\
\hline Frutas (rac/día) & 3 & 2 & 2 & $2-3$ & 3 \\
\hline Lácteos (rac/día) & 3 & $\begin{array}{c}1 \\
\text { En paciente } \\
\text { hipercatabólico: } 7\end{array}$ & 1 & 1,5 & 3 \\
\hline Aceite de Oliva (rac/día) & 6 & 6 & 7 & 6 & 6 \\
\hline $\begin{array}{l}\text { Carnes magras, } \\
\text { carnes blancas pescados, } \\
\text { huevos y legumbres } \\
\text { (rac/día) }\end{array}$ & 2 & $\begin{array}{c}1 \\
\text { En paciente } \\
\text { hipercatabólico:2 }\end{array}$ & $\begin{array}{c}2 \\
\text { En paciente } \\
\text { hipercatabólico:3 }\end{array}$ & 3 & $\begin{array}{c}1 \\
\text { En paciente } \\
\text { hipercatabólico:2 }\end{array}$ \\
\hline Frutos secos (rac/día) & 1 & Individualizar & No recomendado & Individualizar & 1 \\
\hline Azúcares & Moderado & Moderado & Moderado & Muy moderado & Moderada \\
\hline $\begin{array}{l}\text { Dulces, Bollería, } \\
\text { grasas untables } \\
\text { y snacks salados }\end{array}$ & Ocasional & Ocasional & Ocasional & Ocasional & Ocasional \\
\hline
\end{tabular}

Nota: Las raciones han sido calculadas para un peso estimado de $70 \mathrm{~kg}$. Abreviaturas: rac/día, ración/día.

- Monitorizar la ingesta hospitalaria según protocolo de cada hospital, y el riesgo de disfagia.

- Asegurar la adaptación de la dieta, según vaya evolucionando la enfermedad, prestando especial atención a los posibles efectos secundarios de la infección por SARS-CoV-2.

- Seguimiento según práctica clínica habitual en cuanto a administración de fármacos y toma de constantes vitales.

Si mediante la alimentación no se pueden cubrir los requerimientos proteicos o energéticos, se recomienda la utilización de soporte nutricional, ya sea por vía oral o enteral, y seguir las siguientes recomendaciones ${ }^{16}$ :

- En paciente con nutrición enteral por sonda, el cabezal de la cama debe estar siempre elevado a $45^{\circ}$.

- Observar la presencia de síntomas gastrointestinales como diarrea, náuseas o vómitos durante el consumo de la nutrición enteral oral o por sonda.

- Realizar una adecuada progresión de la dieta enteral a la vía oral.

En el caso de pacientes con infección grave por SARSCoV-2, requieren nutrición enteral oral o parental que será prescrita por el Servicio de Nutrición y Endocrinología.
Recomendaciones dietéticas para pacientes con ERC y post-infección por SARS-CoV-2

Las recomendaciones dietéticas en el proceso post infección por SARS-CoV-2 se han desarrollado según el grado de afectación del estado nutricional: riesgo de desnutrición o desnutrición leve (Tabla 4) y desnutrición moderada o grave (Tabla 5). Para la valoración del estado nutricional en la etapa post-infección se recomienda realizar una valoración nutricional completa siguiendo el método de referencia de cada centro, e incluir valoración de la capacidad funcional.

- Las recomendaciones dietéticas de la afectación post-SARS-CoV-2, son las mismas que en afectación SARS-CoV-2-moderada. Debido a que los síntomas descritos relacionados con el estado nutricional son los mismos.

\section{Discusión}

El actual documento pone de manifiesto la necesidad de las adaptaciones dietéticas en pacientes con ERC e infección por SARS-CoV-2. A pesar de que conocemos la elevada incidencia de malnutrición en $\mathrm{ERC}^{17}$, y en el proceso infeccioso por SARS-CoV-218, así como sus consecuencias; hasta la fecha no hemos encontrado nin- 
Tabla 4. Raciones diarias de ingesta recomendadas según estadio de la ERC en pacientes POST infección por SARS-CoV-2 con riesgo de desnutrición o desnutrición leve.

\begin{tabular}{|c|c|c|c|c|c|}
\hline Grupo de alimentos & ERCA 1-3 & ERCA 3-5 sin diálisis & Hemodiálisis & Diálisis Peritoneal & Trasplante \\
\hline Cereales (rac/día) & 5 & 6 & 5 & 4 & 5 \\
\hline $\begin{array}{l}\text { Verduras y hortalizas } \\
\text { (rac/día) }\end{array}$ & 2 & 2 & 2 & 2 & 2 \\
\hline Frutas (rac/día) & 3 & 2 & 2 & $2-3$ & 3 \\
\hline Lácteos (rac/día) & 1,5 & 1,5 & 1 & 1 & 1,5 \\
\hline Aceite de Oliva (rac/día) & 6 & 6 & 6 & 5 & 6 \\
\hline $\begin{array}{l}\text { Carnes, pescados, } \\
\text { huevos y legumbres } \\
\text { (rac/día) }\end{array}$ & 1,5 & 1 & 2 & 2,5 & 1,5 \\
\hline Frutos secos (rac/día) & 1 & & & Individualizar & Individualizar \\
\hline Azúcares & Moderado & Moderado & Moderado & No recomendado & Moderado \\
\hline $\begin{array}{l}\text { Dulces, Bollería, } \\
\text { grasas untables y } \\
\text { snacks salados }\end{array}$ & Ocasional & Ocasional & Ocasional & Ocasional & Ocasional \\
\hline
\end{tabular}

Nota: Las raciones han sido calculadas para un peso estimado de $70 \mathrm{~kg}$. Abreviaturas: rac/día, ración/día.

Tabla 5. Raciones diarias de ingesta recomendadas según estadio de la ERC en pacientes POST infección por SARS-CoV-2 con desnutrición moderada o grave.

\begin{tabular}{|c|c|c|c|c|c|}
\hline Grupo de alimentos & ERCA 1-3 & ERCA 3-5 sin diálisis & Hemodiálisis & Diálisis Peritoneal & Trasplante \\
\hline Cereales (rac/día) & 6 & 7 & 6 & 5 & 6 \\
\hline $\begin{array}{l}\text { Verduras y hortalizas } \\
\text { (rac/día) }\end{array}$ & 2 & 2 & 2 & 2 & 2 \\
\hline Frutas (rac/día) & 3 & 2 & 2 & 3 & 3 \\
\hline Lácteos (rac/día) & 3 & 2 & 1 & 1,5 & 3 \\
\hline Aceite de Oliva (rac/día) & 6 & 6 & 7 & 6 & 6 \\
\hline $\begin{array}{l}\text { Carnes, pescados, } \\
\text { huevos y legumbres } \\
\text { (rac/día) }\end{array}$ & 2 & 2 & 2,5 & 3 & 2 \\
\hline Frutos secos (rac/día) & 1 & Individualizar & No recomendado & Individualizar & 1 \\
\hline Azúcares & Moderado & Moderado & Moderado & No recomendado & Moderado \\
\hline $\begin{array}{l}\text { Dulces, Bollería, } \\
\text { grasas untables y } \\
\text { snacks salados }\end{array}$ & Ocasional & Ocasional & Ocasional & Ocasional & Ocasional \\
\hline
\end{tabular}

Nota: Las raciones han sido calculadas para un peso estimado de $70 \mathrm{~kg}$. Abreviaturas: rac/día, ración/día.

gún estudio que evalúe el tratamiento dietético en este colectivo de pacientes.

Consideramos al igual que otros autores ${ }^{19,20}$, que la valoración precoz del estado nutricional con los métodos disponibles, limitando el contacto físico, y centrada en valorar la presencia de: anorexia, disfagia, disnea, ageusia y diarrea, contribuirá a un mejor tratamiento nutricional, ayudando a la disminución de la prevalencia de malnutrición así como a la peor evolución del proceso de malnutrición y de infección por SARS-CoV-2.

Casi la totalidad de pacientes con infección moderada-grave por SARS-CoV-2 presentan un grado de inflamación elevada ${ }^{21}$, siendo otro punto a considerar en el abordaje dietético de este colectivo de pacientes al 
verse incrementados sus requerimientos proteicos y energéticos independientemente de la funcionalidad renal, por lo que consideramos al igual que en procesos de infección aguda que es recomendable ajustar las recomendaciones dietéticas, y si estas no son suficientes comenzar precozmente con la utilización de suplementación oral ${ }^{22}$.

El prevalencia de sarcopenia en ERC es elevada, aumenta con la edad y la inflamación ${ }^{23}$, condiciones dadas en la mayoría de los pacientes con infección por SARSCoV-2, y también descritas en pacientes con infección SARS-CoV2 sin ERC ${ }^{24}$, por lo que recomendamos su valoración y seguimiento, siendo uno de los elementos claves en la valoración del paciente post-infección.

Consideramos que una vez pasado el cuadro de activo de infección, todos los pacientes deben ser monitorizados mediante una valoración nutricional completa, en la que se debe incluir la valoración de la sarcopenia y seguir todas las modificaciones dietéticas prescritas, así como sus adaptaciones. Debido a que según nuestro criterio y el de la literatura consultada ${ }^{25}$ es el momento de mayor importancia para revertir la situación de malnutrición y sus consecuencias.

La principales limitaciones del presente documento son la poca evidencia disponible en el paciente con ERC e infección por SARS-CoV-2, y la dificultad en unificar las recomendaciones del proceso infeccioso con el tratamiento dietético del paciente con ERC, sin aumentar las restricciones y cubriendo los requerimientos en cada fase de infección.

A modo de conclusión podemos decir que en los pacientes con ERC e infección por SARS-CoV-2 es recomendable la monitorización del estado nutricional, mediante la utilización de herramientas en las que se minimice el contacto físico. Es importante adaptar las recomendaciones dietéticas no solo al estadio de la enfermedad renal sino también a la etapa de infección por SARSCoV-2, prestando especial atención a la presencia de disnea, disfagia, anorexia, hipogeusia y diarrea para la modificación de las recomendaciones dietéticas.

(El presente artículo está basado en el Consenso Multidisciplinar sobre la valoración y el tratamiento nutricional y dietético en pacientes con Enfermedad Renal Crónica e infección por SARS-CoV-2)
Recibido: 21-07-2020

Revisado: 28-07-2020

Modificado 08-08-2020

Aceptado: 26-08-2020

\section{Bibliografía}

1. Cheng Y, Luo R, Wang K, Zhang M, Wang Z, Dong L, et al. Kidney impairment is associated with in-hospital death of COVID-19 patients. MedRxiv 2020: Feb. [En prensa].

2. Li Z, Wu M, Yao J, Guo J, Liao X, Song S, et al. Caution on Kidney Dysfunctions of COVID-19 Patients. MedRxiv 2020 Feb. [En prensa].

3. Wang D, Hu B, Hu C, Zhu F, Liu X, Zhang J, et al. Clinical Characteristics of 138 Hospitalized Patients With 2019 Novel Coronavirus-Infected Pneumonia in Wuhan, China. JAMA. 2020;323:1061-9.

4. Chen N, Zhou M, Dong X, Qu J, Gong F, Han Y, et al. Epidemiological and clinical characteristics of 99 cases of 2019 novel coronavirus pneumonia in Wuhan, China: a descriptive study. The Lancet. 2020;395:507-13.

5. Guan W, Ni Z, Hu Y, Liang W, Ou C, He J, et al. Clinical Characteristics of Coronavirus Disease 2019 in China. N Engl J Med. 2020; 382:1708-20.

6. Barazzoni R, Bischoff SC, Breda J, Wickramasinghe K, Krznaric Z, Nitzan D, et al. ESPEN expert statements and practical guidance for nutritional management of individuals with SARS-CoV-2 infection. Clinical Nutrition. 2020;39:1631-8.

7. Cederholm T, Jensen GL, Correia MITD, Gonzalez MC, Fukushima R, Higashiguchi T, et al. GLIM criteria for the diagnosis of malnutrition - $A$ consensus report from the global clinical nutrition community. Clin Nutr. 2019;38:1-9.

8. Malmstrom TK, Morley JE. SARC-F: A Simple Questionnaire to Rapidly Diagnose Sarcopenia. J Am Med Dir Assoc. 2013;14(8):531-2.

9. Aranceta J, Arija V, Maíz E, Martinez E, Ortega R, Pérez-Rodrigo Cetal. Guías alimentarias para la poblaciónespañola (SENC, 2016); la nueva pirámide de la alimentación saludable. Nutr Hosp. 2016;33(Supl. 8):1-48. 
10. Wright M, Southcott E, MacLaughlin H, Wineberg S. Clinical practice guideline on undernutrition in chronic kidney disease. BMC Nephrol. 2019;20:370.

11. Clinical Practice Guideline for Nutrition chronic Kidney Disease: 2019 UPDATE. National Kidney Foundaiton NKF). [Consultado 2 May 2020]. Disponible en: https://www.kidney.org/sites/default/files/ Nutrition_GL\%2BSubmission_101719_Public_Review_Copy.pdf.

12. Jin $Y-H$, Cai L, Cheng Z-S, Cheng H, Deng T, Fan Y-P, et al. $A$ rapid advice guideline for the diagnosis and treatment of 2019 novel coronavirus (2019-nCoV) infected pneumonia (standard version). Mil Med Res. 2020;7:4.

13. Kidney Disease: Improving Global Outcomes (KDIGO) CKD-MBD Update Work Group. KDIG0 2017 Clinical Practice Guideline Update for the Diagnosis, Evaluation, Prevention, and Treatment of Chronic Kidney Disease-Mineral and Bone Disorder (CKDMBD). Kidney Int Suppl (2011). 2017;7:1-59.

14. Posición de expertos sobre el manejo nutricional del coronavirus COVID 19. Comité Internacional para la Elaboración de Consensos y Estandarización en Nutriología (CIENUT). Abril 2020. [Consultado 2 May 2020]. Disponible en: https://cienut.org/comite_internacional/declaraciones/pdf/declaracion2.pdf.

15. Lomax AR, Calder PC. Prebiotics, immune function, infection and inflammation: a review of the evidence. Br J Nutr. 2009; 101:633-58.

16. Zhang L, Liu Y. Potenial al interventions for novel coronavirus in China: a systematic review. J Med Virol. 2020; 92:479-90.

17. Hyun YY, Lee KB, Han SH, Kim YH, Kim YS, Lee SW, et al. Nutritional Status in Adults with Predialysis Chronic Kidney Disease: KNOW-CKD Study. J Korean Med Sci. 2017 Feb;32(2):257-63.
18. Tao Li, Yalan Zhang, Cheng Gong, Jing Wang, Bao Liu, Li Shi and Jun Duan Eur J Clin Nutr. 2020;74(6):871-5.

19. Kalantar-Zadeh and Moore. Impact of Nutrition and Diet on COVID-19 Infection and Implications for Kidney Health and Kidney Disease Management. J Ren Nutr. 2020;30(3):179-81.

20. Handu D, Moloney L, Rozga M, Cheng F. Malnutrition Care during the COVID-19 Pandemic: Considerations for Registered Dietitian Nutritionists Evidence Analysis Center. J Acad Nutr Diet. 2020 may. [En prensa].

21. Iddir M, Brito A, Dingeo G, Fernandez Del Campo SS, Samouda $H$, et al. Strengthening the Immune System and Reducing Inflammation and Oxidative Stress through Diet and Nutrition: Considerations during the COVID-19 Crisis. Nutrients. 2020;12(6):1562.

22. Caccialanza R, Laviano $A$, Lobascio F, Montagna $E$, Bruno R, Ludovisi $S$, et al. Early nutritional supplementation in non-critically ill patients hospitalized for the 2019 novel coronavirus disease (COVID-19): Rationale and feasibility of a shared pragmatic protocol. Nutrition 2020 abr. [En prensa].

23. Watanabe H, Enoki Y, Maruyama T. Sarcopenia in Chronic Kidney Disease: Factors, Mechanisms, and Therapeutic Interventions. Biol Pharm Bull. 2019; 42(9):1437-45.

24. Morley JE, Kalantar-Zadeh K, Anker SD. COVID-19: a major cause of cachexia and sarcopenia? J Cachexia Sarcopenia Muscle. 2020 Aug; 11(4): 863-5.

25. Rovere Querini P, De Lorenzo R, Conte $C$, et al. Post-COVID-19 follow-up clinic: depicting chronicity of a new disease. Acta Biomed. 2020;91(9-S):22-8.

Este artículo se distribuye bajo una Licencia Creative Commons Atribución-NoComercial 4.0 Internacional. https://creativecommons.org/licenses/by-nc/4.0/

Open Access (C) (1) (8) 